\title{
Structure of the yeast histone H3-ASF I interaction: implications for chaperone mechanism, species-specific interactions, and epigenetics
}

\author{
Andrew J Antczak ${ }^{1}$, Toshiaki Tsubota ${ }^{2}$, Paul D Kaufman*2 and \\ James M Berger*1
}

Address: ${ }^{1}$ Department of Molecular and Cell Biology, University of California, Berkeley, California 94720, USA and ${ }^{2}$ Program in Gene Function and Expression, University of Massachusetts Medical School, Worcester, Massachusetts 01605, USA

Email: Andrew J Antczak - antczaka@berkeley.edu; Toshiaki Tsubota - Toshiaki.Tsubota@umassmed.edu;

Paul D Kaufman* - paul.kaufman1@umassmed.edu; James M Berger* - jmberger@berkeley.edu

* Corresponding authors

Published: 13 December 2006

BMC Structural Biology 2006, 6:26 doi:10.1 186/1472-6807-6-26

This article is available from: http://www.biomedcentral.com/I472-6807/6/26

(c) 2006 Antczak et al; licensee BioMed Central Ltd.

This is an Open Access article distributed under the terms of the Creative Commons Attribution License (http://creativecommons.org/licenses/by/2.0), which permits unrestricted use, distribution, and reproduction in any medium, provided the original work is properly cited.

\begin{abstract}
Background: The histone $\mathrm{H} 3 / \mathrm{H} 4$ chaperone Asfl (anti-silencing function I) is required for the establishment and maintenance of proper chromatin structure, as well as for genome stability in eukaryotes. Asfl participates in both DNA replication-coupled (RC) and replication-independent (RI) histone deposition reactions in vitro and interacts with complexes responsible for both pathways in vivo. Asfl is known to directly bind histone $\mathrm{H} 3$, however, high-resolution structural information about the geometry of this interaction was previously unknown.
\end{abstract}

Results: Here we report the structure of a histone/histone chaperone interaction. We have solved the $2.2 \AA$ crystal structure of the conserved $\mathrm{N}$-terminal immunoglobulin fold domain of yeast Asfl (residues 2-155) bound to the C-terminal helix of yeast histone $\mathrm{H} 3$ (residues I2I-134). The structure defines a histone-binding patch on Asfl consisting of both conserved and yeastspecific residues; mutation of these residues abrogates $\mathrm{H} 3 / \mathrm{H} 4$ binding affinity. The geometry of the interaction indicates that Asfl binds to histones $\mathrm{H} 3 / \mathrm{H} 4$ in a manner that likely blocks sterically the $\mathrm{H} 3 / \mathrm{H} 3$ interface of the nucleosomal four-helix bundle.

Conclusion: These data clarify how Asfl regulates histone stoichiometry to modulate epigenetic inheritance. The structure further suggests a physical model in which Asfl contributes to interpretation of a "histone $\mathrm{H} 3$ barcode" for sorting $\mathrm{H} 3$ isoforms into different deposition pathways.

\section{Background}

Chromatin structure plays a critical role in all processes involving the genome of eukaryotic organisms. The first step in chromatin establishment de novo is the deposition of an $(\mathrm{H} 3 / \mathrm{H} 4)_{2}$ heterotetramer onto DNA by one or more of several histone chaperones including Asf1, CAF-1, and the HIR Complex. The pre-nucleosomal $(\mathrm{H} 3 / \mathrm{H} 4)_{2}$ intermediate then binds to two histone $\mathrm{H} 2 \mathrm{~A} / \mathrm{H} 2 \mathrm{~B}$ heterodimers, which complete the core histone octamer and allow for proper wrapping of nucleosomal DNA (reviewed in [1]). 
Histone $\mathrm{H} 3 / \mathrm{H} 4$ chaperones participate in two similar, yet functionally distinct pathways. The first involves replication-coupled (RC) histone deposition during S-phase, in which CAF-1-histone complexes are recruited to sites of DNA replication through an interaction with the DNA polymerase processivity factor PCNA [2]. The second pathway is a replication-independent (RI) histone deposition process that occurs during all stages in the cell cycle and involves the HIR Complex [3-5]. RI histone deposition has been linked to chromatin reorginization during both transcription [6] and embryogenesis $[7,8]$.

Most multicellular eukaryotes, unlike fission and budding yeasts, contain two major types of histone $\mathrm{H} 3$ isoforms: the very closely related H3.1/H3.2 proteins, and the more distinct H3.3 variant [9]. Although these histones differ by only a few amino acids, expression and deposition of H3.1/H3.2 is limited to S phase, while H3.3 is expressed and deposited at all times during the cell cycle [10]. Consistent with CAF-1's role in RC deposition, CAF-1 has been purified from human cell extracts in complex with histone H3.1 but not H3.3 [11]. Conversely, HIRA, the human homologue of yeast Hir1 and Hir2, can be found complexed with H3.3 but not H3.1 [11].

Asf1 is the common entity shared by the RC and RI pathways, because it binds to and stimulates histone deposition by both the CAF-1 and HIR complexes [4,11-14]. Asf1 proteins from all eukaryotes share a very conserved 155 residue $\mathrm{N}$-terminal immunoglobulin-like fold domain [15]. In contrast, the C-termini of Asf1 proteins from different organisms are highly divergent. Interestingly, DmAsf1, HsAsf1a, and HsAsf1b share nearly $60 \%$ sequence identity within the conserved $\mathrm{N}$-terminal domain, yet all fail to fully restore DNA damage resistance or chromatin-mediated gene silencing in yeast when placed under the control of the endogenous promoter [16]. These data suggest that species-specific interactions within this domain are functionally crucial. Notably, the yeast Asf1 N-terminal domain is fully functional as a truncation both in vitro $[15,17]$ and in vivo $[15,18,19]$.

To date, structural information on how histone chaperones associate with histones and/or each other has been sparse. To gain insight into histone deposition, we have employed structural, biochemical, and genetic techniques to study the interaction between Asf1 and histones H3/ $\mathrm{H} 4$. We determined the structure of the conserved N-terminus of budding yeast Asf 1 bound to the C-terminal $\alpha 3$ helix of yeast histone $\mathrm{H} 3$ to 2.2 Å resolution. This peptide was chosen because it was shown to interact with Asf1 through NMR experiments [20] as well as two-hybrid analysis [21]. In addition, this helix is the known site of $\mathrm{H} 3-\mathrm{H} 3$ interactions in the core tetramer of the nucleosomal octamer, and most likely is involved in the regula- tion of histone stoichiometry. The structure identifies several residues that are critical for the interaction, and we demonstrate that mutation of these residues affects histone $\mathrm{H} 3 / \mathrm{H} 4$ binding in vitro and causes characteristic silencing phenotypes in vivo. Remarkably, modeling of this structure into the histone core octamer of the nucleosome indicates that binding of the conserved $\mathrm{N}$-terminus of Asf 1 to H3 directly occludes H3/H3 homodimer interactions. This work therefore provides detailed information about the interaction between a histone chaperone protein and client histone target. These data also provide insights into how Asf1 affects both the inheritance of chromatin structures and the deposition of different histone $\mathrm{H} 3$ isoforms.

\section{Results \\ Structure of AsfIN bound to $H 3 \alpha 3$}

The conserved N-terminus of yeast Asf1 (residues 2-155, herein referred to as Asf $1 \mathrm{~N}$ ) is sufficient for wild type function in vivo, and binds histones H3/H4, RFC, and Rad53 in vitro $[15,17,18]$. Because the interaction between the $\mathrm{C}$ terminal histone $\mathrm{H} 3 \alpha 3$ helix $(\mathrm{H} 3 \alpha 3)$ and Asf1 is of modest affinity (micromolar $\mathrm{K}_{\mathrm{d}}$ ) [20], we stabilized the association by fusing Asf $1 \mathrm{~N}$ to $\mathrm{H} 3 \alpha 3$ with a flexible linker peptide. The fusion protein was overexpressed in E. coli, purified to homogeneity, and used for crystallization studies (see Methods).

We determined the structure of Asf1N bound to $\mathrm{H} 3 \alpha 3$ to $2.2 \AA$ resolution, using a previously solved structure of yeast Asf1N for molecular replacement [15]. After several rounds of refinement using a histone-free model of Asf1, density corresponding to the histone helix became clearly visible, permitting manual building of the H3 helix. Simulated annealing omit maps [22] further confirmed the electron density and model built for this region (Figure 1A). As seen previously, Asf1 alone adopts a switched-type immunoglobulin-like (Ig-like) fold containing two extra, short $\beta$-strands (termed $h$ and $h '$, Figure $1 \mathrm{~A}$ ).

The global fold of Asf $1 \mathrm{~N}$ in the current structure is nearly identical to that solved previously without the histone helix (Figure 1B). The only significant changes in the structure are a forward rotation of the loop connecting $\beta$ strands c and c', as well as a small rotation of the loop and $\alpha$-helix connecting $\beta$-strands $e$ and $f$. Interestingly, the positions of both of these regions resemble those found in the NMR structure of the human homologue HsAsf1aN (Figure 1B) [20]. These observations suggest that specific regions of Asf1 $\mathrm{N}$ may adopt multiple local conformations depending upon its binding partner.

\section{The histone binding pocket on AsfI}

NMR experiments have shown that a short C-terminal H3 peptide (residues 122-137) adopts its proper helical 
A
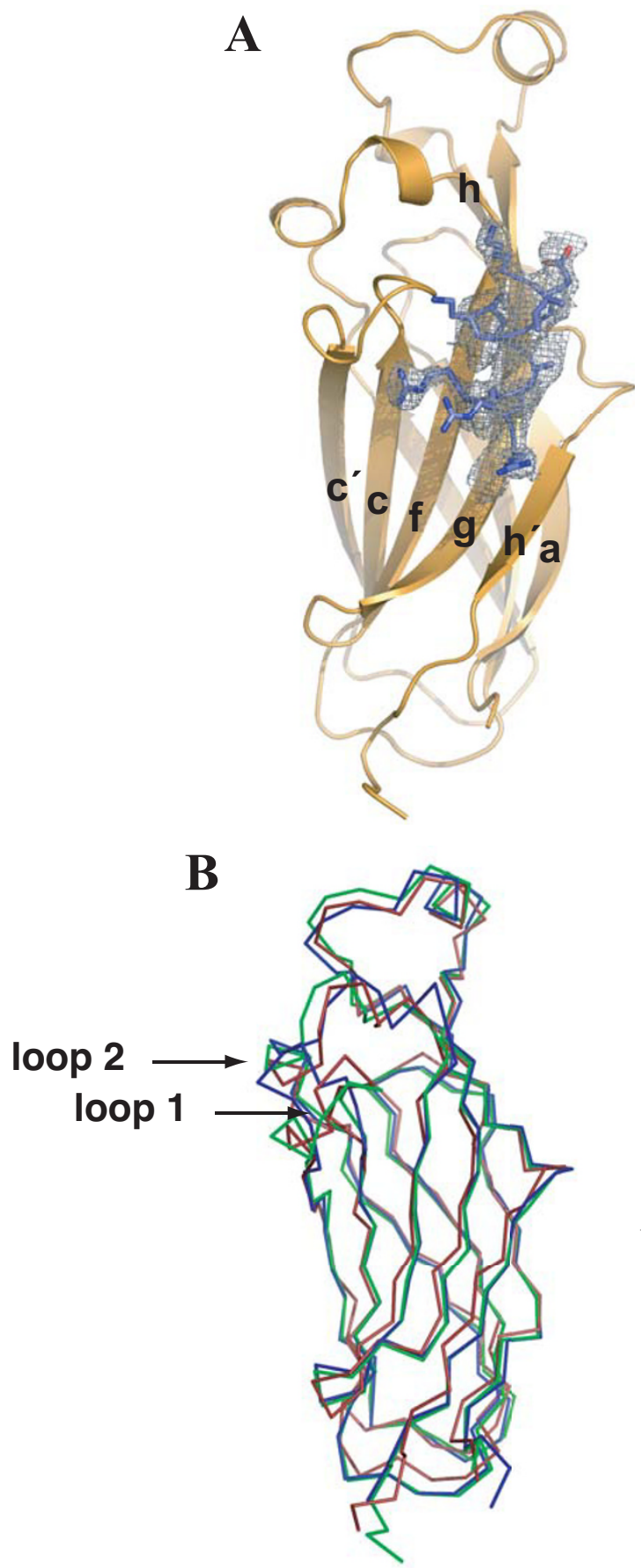

"Front"
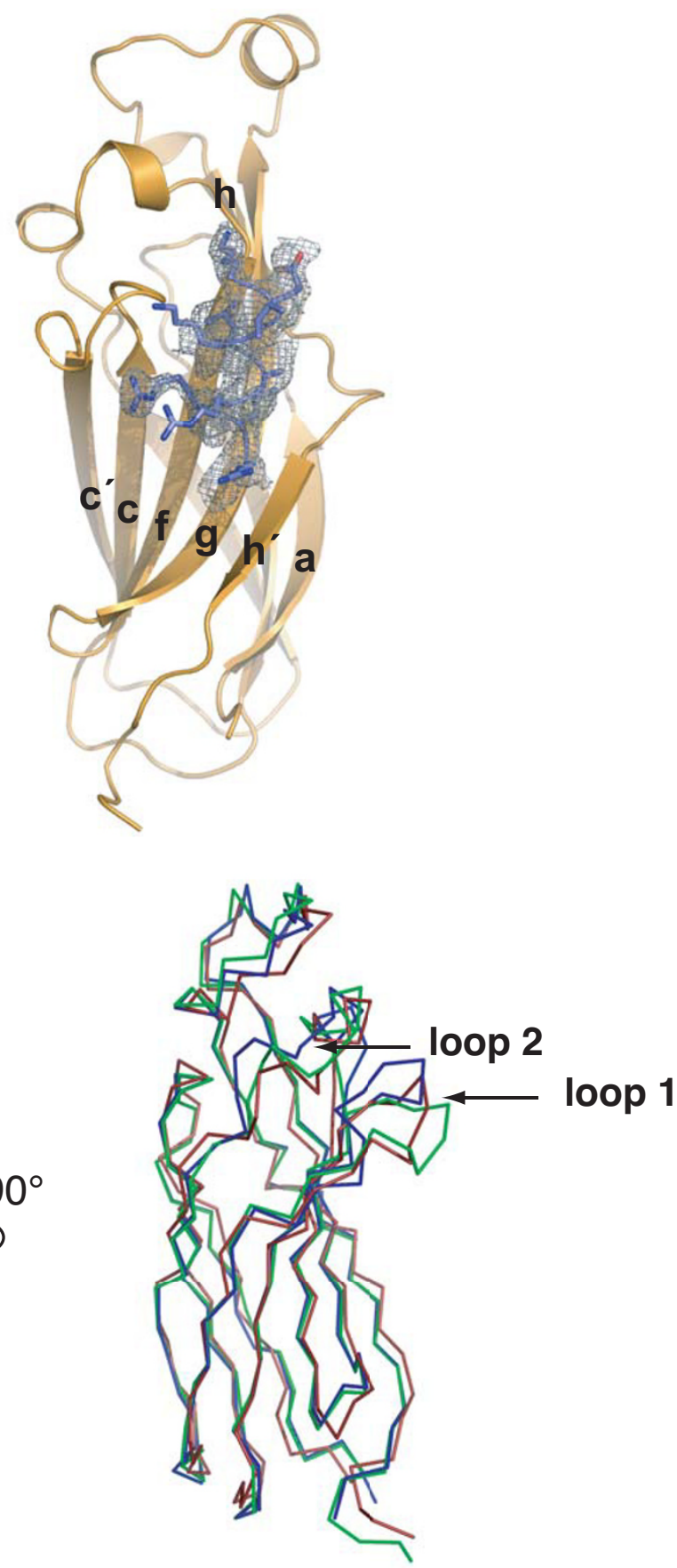

"Side"

\section{Figure I}

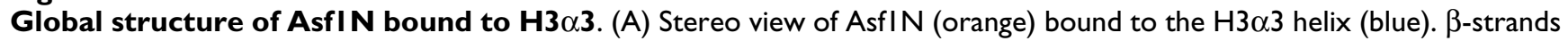
are labeled as per the nomenclature for switched-type lg-like folds. A section of an electron density omit-map generated in the absence of the histone helix is shown contoured at $2.5 \%$. (B) Front and side views superposing known Asfl structures. $\alpha$-carbon atoms of Asfl from the current structure (green), the non-histone bound yeast AsfI (PDB ID IROC, blue), and the human Asfla (PDB ID ITEY, red) were used for the alignment and shown as a-carbon representations. Arrows indicate the loops connecting b-strands $\mathrm{c}$ and $\mathrm{c}^{\prime}$ (loop $\mathrm{I}$ ), and connecting b-strands e and $\mathrm{f}$ (loop 2), which both rotate forward upon binding $\mathrm{H} 3 \alpha 3$. 
structure in the presence but not absence of $H s A s f 1 a N$ [20]. The same study further identified a probable histone-binding patch on the front $\beta$-sheet of Asf $1 \mathrm{~N}$ using genetic and biochemical analyses of mutant proteins. These studies did not identify, however, the geometry of the interaction nor the complete retinue of specific histone-Asf1 interactions.

The structure solved here shows that the histone helix binds to Asf1 in this predicted region, but in an unanticipated orientation. Mutational analysis of this region [20] had led to the prediction that the helix axis would run perpendicular to $\beta$-strand direction of Asf1, as opposed to the parallel configuration we now confirm in our structure. The H3 $\alpha$-helix lies in a pocket defined by hydrophobic residues on one side and an intricate hydrogen-bonding network on the other (Figure 2). Notably, to properly form the pocket, the $\beta-c / \beta-c^{\prime}$ connecting loop folds downward (as compared to the structure of unbound yeast Asf1N), bringing residues Ser 48 and Ser50 in proximity to contribute to the hydrogen-bonding network ("loop 1" in Figure $1 \mathrm{~B})$.

The residues comprising the hydrophobic portion of the interface between both Asf 1 and $\mathrm{H} 3$ are essentially identical throughout all eukaryotic species sequenced to date $[9,15]$. The one exception to this rule is Leu130 of $\mathrm{H} 3$ in S. cerevisiae, which is changed to the similar amino acid isoleucine in all other eukaryotes. Leu130 makes van der Waals contacts to Asf1 amino acids Val94, Tyr112, Arg145, and Leu96 (Figure 3A). Leu126 of histone H3 also makes hydrophobic interactions with Val92 and Val94, both of which are well-conserved residues on Asf1.

A lone salt bridge is formed between the invariant residues Asp54 on Asf1 and Arg129 on H3. The carboxylate and guanidinium groups on these side chains sit approximately $3 \AA$ apart and further coordinate a pair of water molecules (Figure 2). Interestingly, these waters participate in an extensive hydrogen-bond network (Figure 3A) that includes a polar interaction between the invariant Lys122 on H3 and the yeast-specific Asf1 residue Ser91, as well as the main chain carbonyl of its neighboring amino acid, Val92. The two water networks that coordinate the interactions described above are bridged by a second yeast-specific residue, Ser48, which hydrogen-bonds to waters in both networks. The yeast-specific residues central to the hydrogen-bonded network suggest that this interaction may comprise a species-specificity determinant (Figure 3B, see Discussion). Lastly, Asf1 residue Thr147 and the main chain carbonyl from H3-L130 interact indirectly by hydrogen bonding to a bridging water molecule. Importantly, no density was seen for the eight amino acid linker peptide past the first two alanine residues, indicating that the fusion has not constrained the

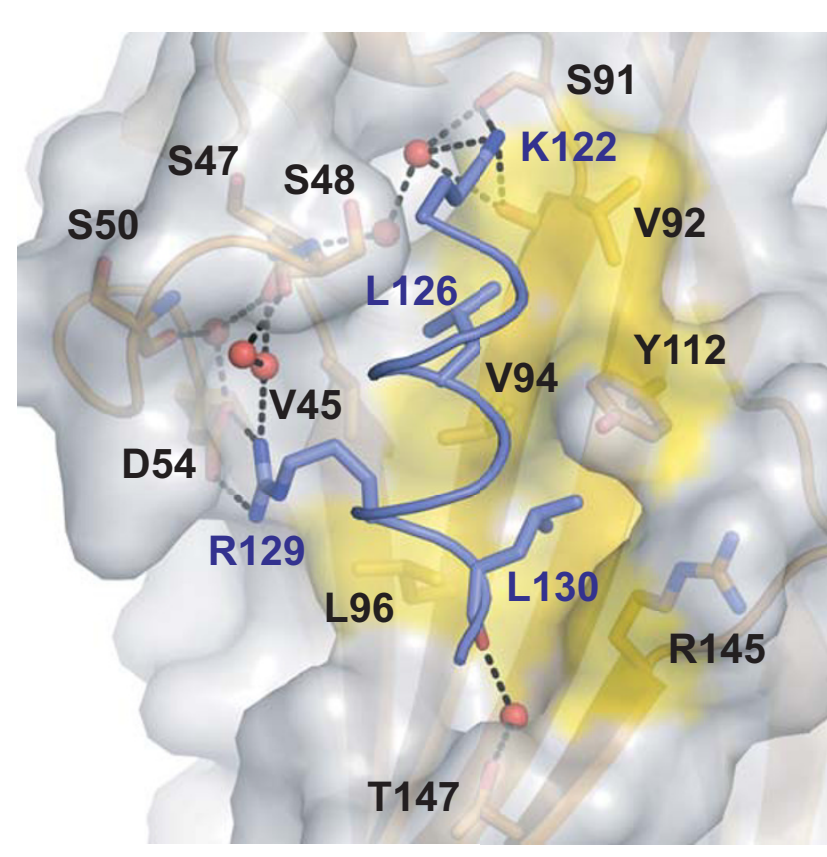

Figure 2

Close-up view of the histone-binding patch on Asfl. Residues shown to be important for the interaction are labeled and represented as sticks, with labels in black for Asfl and blue for histone $\mathrm{H} 3$ residues. Waters are represented as red spheres, hydrogen bonds as black dashed lines, and the hydrophobic surface participating in van der Waals interactions is shaded yellow.

orientation of the H3/Asf1 $\mathrm{N}$ interaction in an artificial manner.

\section{Genetic analysis of histone binding mutants}

To assess the in vivo relevance of the contacting residues identified by our structure, we examined heterochromatic gene silencing in Asf1 mutant cells, because Asf1 is crucial for silencing in yeast cells lacking $C A C$ genes encoding CAF-1 subunits $[12,13]$. We therefore expressed wild-type and mutant ASF1 genes in a cac1 $1 \Delta a s f 1 \Delta$ strain and measured silencing of a URA3 reporter gene at the left telomere of chromosome VII.

In line with previous reports [13], cac1 asf1 cells transformed with a wt Asf1-expressing plasmid were able to grow on plates containing 5-FOA (a lethal substrate for cells expressing the Ura3 enzyme, Figure 4), indicating that telomeric silencing is stimulated by functional Asf 1 protein. As a negative control, cells carrying an empty vector could not grow on 5-FOA plates, indicating defective silencing. As another control, the mutations H36A/D37A, residues important for Asf1's interaction with HIRA and Cac2 $[15,23]$, as well as the proximal mutations H39A/ $\mathrm{K} 41 \mathrm{~A}$, caused the expected defective silencing phenotypes. 
A

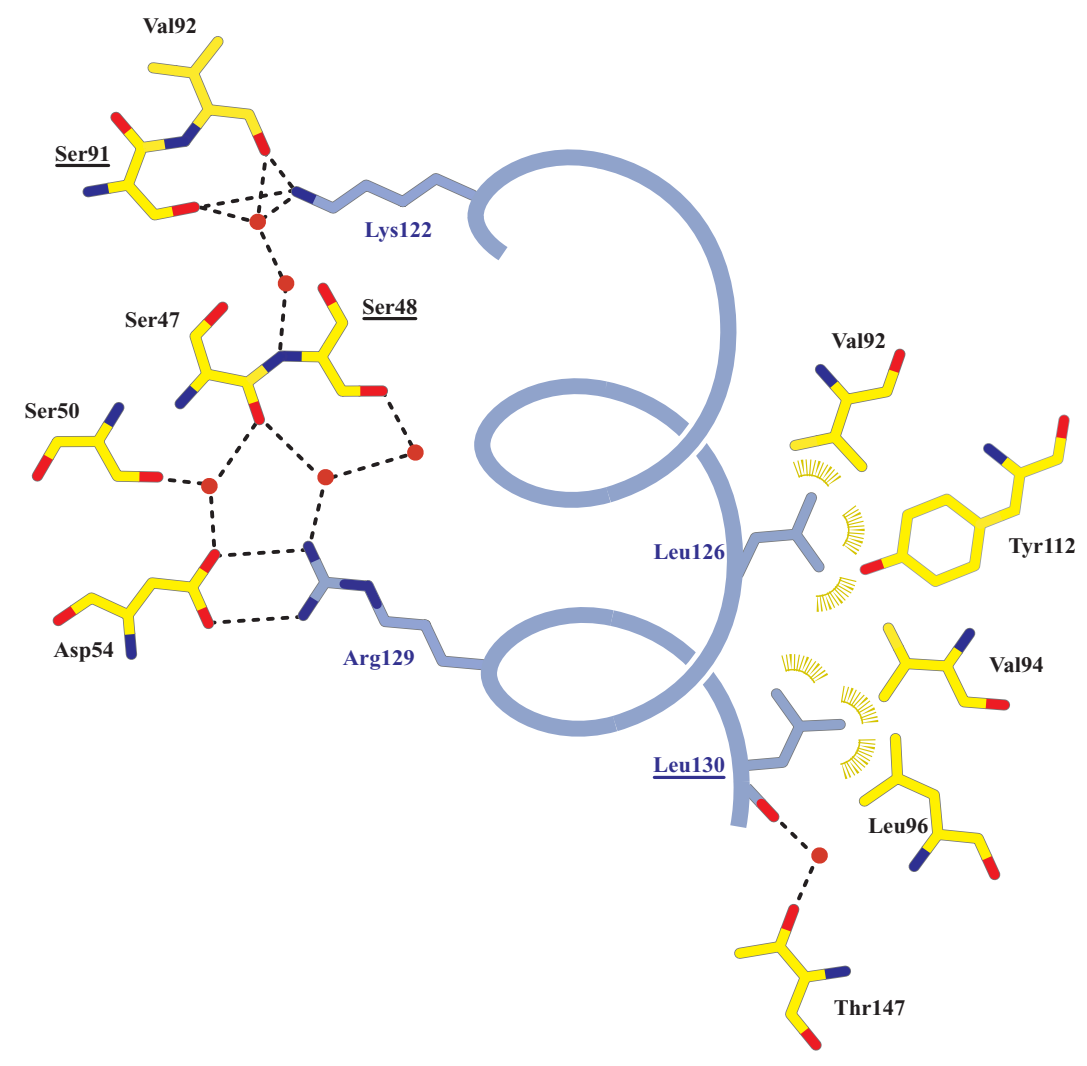

B

Asf1

$\underline{\mathrm{H} 3}$

C. elegans

A. gambiae

D. melanogaster

H. Sapiens

O. sativa

B. oleracea

N. crassa

S. pombe

S. cerevisiae

L. infantum

T.brucei

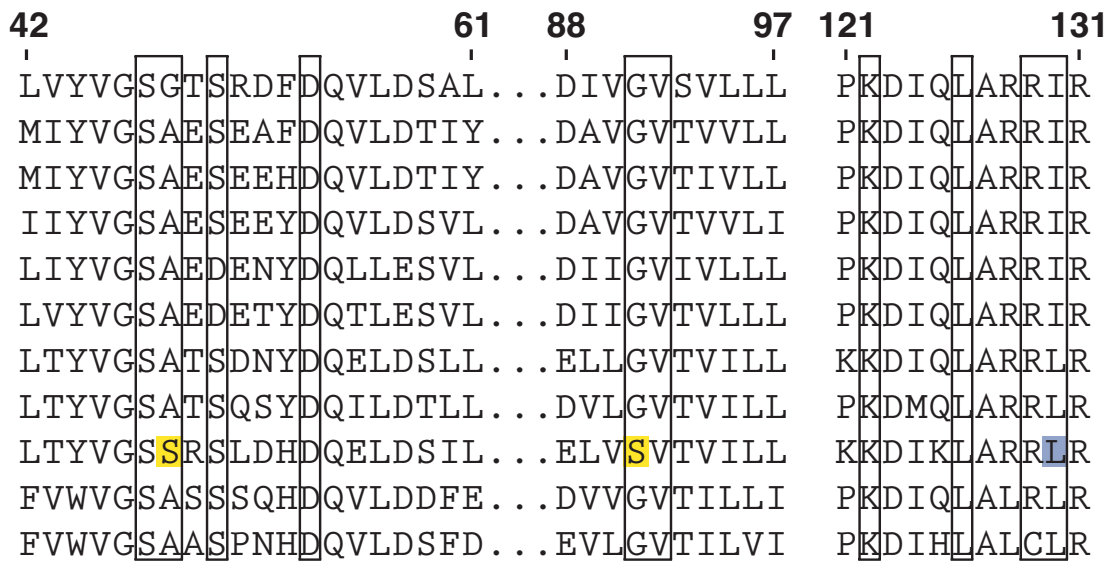

\section{Figure 3}

Structural details of the Asf IN-H3a3 interface. (A) Schematic of the interactions between Asfl and $\mathrm{H} 3 \alpha 3$. Asfl residues are displayed in yellow, $\mathrm{H} 3 \alpha 3$ in blue. Yeast-specific residues on Asf I and $\mathrm{H} 3 \alpha 3$ are underlined. lon pair and hydrogen bonding interactions measuring $3.3 \AA$ or less are represented as dashed lines. Van der Waals interactions are shown as gold arcs. (B) Alignment of Asfl and $\mathrm{H} 3$ residues involved in the interaction across several eukaryotes. Residues participating in the interaction are boxed, with yeast specific Asfl residues highlighted in yellow and H3-Leu I30 highlighted in blue. ClustalW was used to generate the Asfl alignment. 
In contrast, cells transformed with plasmids containing mutations in residues proximal to, but outside of the histone binding patch, such as N114A/E116A and E142A/ K143A double mutants, displayed robust growth on 5FOA containing plates. Therefore, our assay can distinguish point mutations that specifically affect the silencing function of Asf1.

Notably, cells transformed with plasmids harboring mutations in residues participating in the Asf1-H3 interac- tion identified by our structure were unable to grow on 5FOA-containing media. Specifically, V94D/L96D double mutants displayed poor silencing, as did R145A/T147A and H53A/D54A double mutants. Tyr112 mutations caused similar defective silencing phenotypes. One other residue that is proximal to the interface is Val45; V45D mutants likewise displayed defective silencing, suggesting that the conserved hydrophobic patch that mediates the Asf1/H3 interaction is intolerant for acidic side chains. We thus conclude that defects in heterochromatic gene silenc-

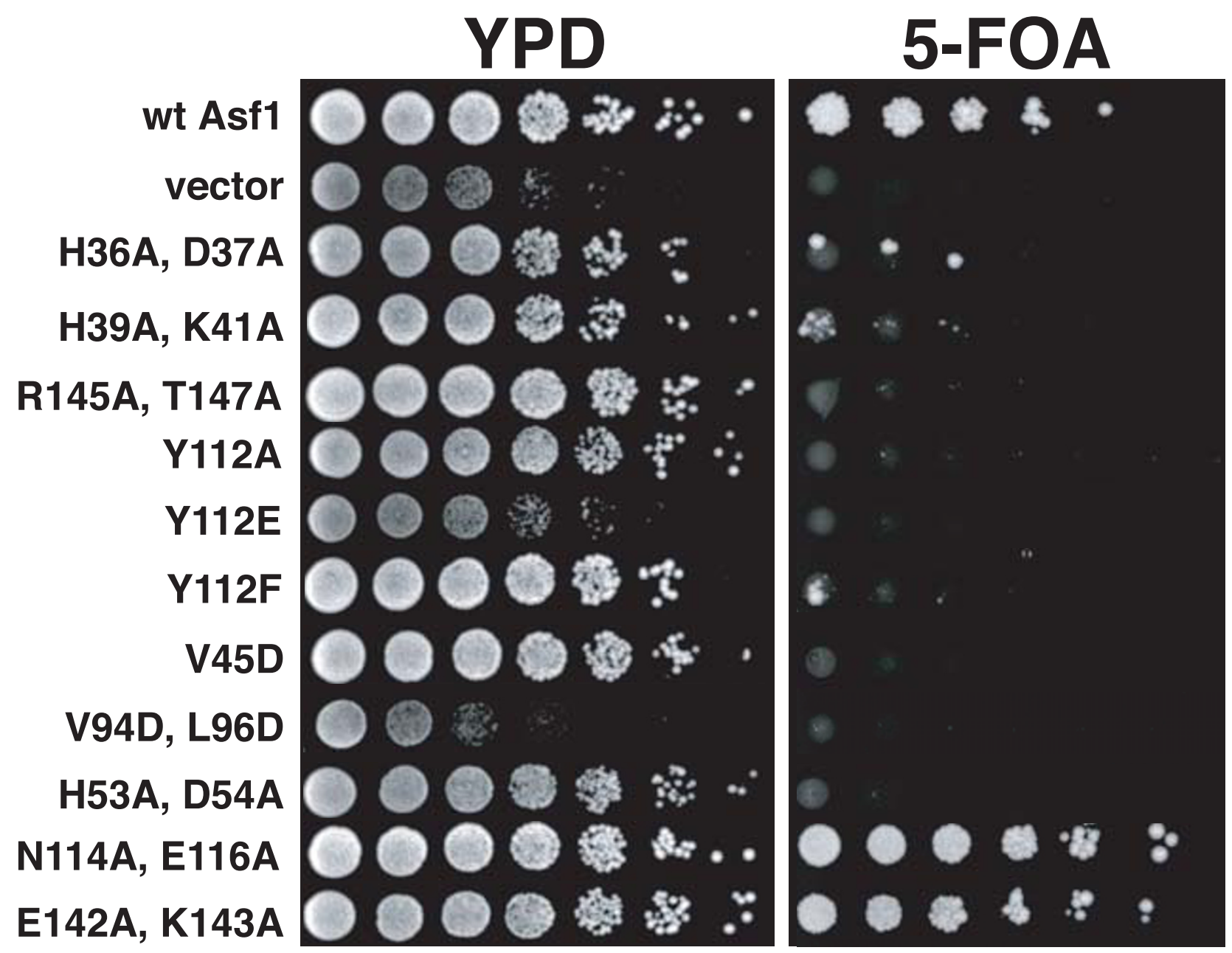

\section{Figure 4}

Genetic analysis of Asf I residues important for histone binding. Asfl histone binding mutants cause silencing defects in vivo. cacl $\Delta$ asfl $\Delta$ cells were transformed with plasmids expressing the indicated Asfl proteins. A four-fold dilution series was spotted onto rich media (YPD) to indicate cell number and onto media containing 5-FOA to measure silencing of a telomeric URA3 reporter gene. 
ing can arise from perturbation of the H3-Asf1 interface contacts identified by our structure.

\section{Biochemical characterization of Asf IN-H3 interactions}

To further validate our structure, we tested mutant Asf1 proteins for binding histones $\mathrm{H} 3 / \mathrm{H} 4$ using a co-precipitation assay of proteins co-expressed in bacteria. As expected, wt Asf1 was able to co-precipitate H3, while pull-downs from extracts containing no Asf1 yielded no H3 (Figure 5). We further observed that Asf1 mutants containing single point changes in residues implicated by our structure (D54A, V94A, and Y112E), as well as the double mutants R145A/T147A and V94D/L96D, failed to co-precipitate H3 (Figure 5A). Importantly, mutant Asf1 proteins with alterations in residues not involved in the Asf1/ H3 interaction, including the N114A/E116A, E39/K41A, and H36A/D37A double mutants, were still able to coprecipitate H3 (Figure 5B).

These results highlight the importance of both the van der Waals and ionic interactions identified here structurally to binding affinity and Asf1 function. Importantly, the L96A and H53A mutations in Asf1, which only weakly perturb histone association (Figure 5A), are seen to be only peripherally involved in $\mathrm{H} 3$ contacts. Together, these data indicate that the Asf1-histone interface revealed by the structural data is physiologically relevant, although we note that our model does not preclude the possibility of additional contacts beyond those observed here arising between Asf1 and other regions of histones $\mathrm{H} 3$ and/or H4.

\section{Asfl is poised to sterically block $\mathrm{H} 3 / \mathrm{H} 4$ heterotetramerization}

Several lines of evidence suggest that histone $\mathrm{H} 3 / \mathrm{H} 4$ chaperones, including Asf1, interact with dimeric and not tetrameric $\mathrm{H} 3 / \mathrm{H} 4$ species $[11,24,25]$. However, histones $\mathrm{H} 3 / \mathrm{H} 4$ spontaneously form tetramers under physiological conditions $[26,27]$. These data indicate that histone chaperones can somehow regulate the propensity of the $\mathrm{H} 3 / \mathrm{H} 4$ heterodimer to tetramerize. To better understand this process, we superposed the Asf1-H3 $\alpha 3$ interaction onto the known $(\mathrm{H} 3 / \mathrm{H} 4)_{2}$ structure of the yeast nucleosome (PDB ID 1ID3) [28] using $\mathrm{H} 3 \alpha 3 \alpha$-carbons from our structure as a guide. Remarkably, inspection of the two structures reveals that Asf1 binds to histone $\mathrm{H} 3$ in an orientation that directly occludes formation of the fourhelix bundle formed at the $\mathrm{H} 3 / \mathrm{H} 3$ dimer interface (Figure $6)$. We discuss the implications of this model below.

\section{Discussion \\ Species-specific differences in the Asfl-H3 interaction}

Although Asf1 and histone H3 are all very highly conserved across Eukaryota, we find that there are several species-specific residues participating in their interaction.
This observation suggests that $S$. cerevisae Asf1 may coordinate its histones somewhat differently than metazoan orthologs of Asf1. Such behavior would help explain why DmAsf1, HsAsf1a, and HsAsf1b all fail to fully restore heterochromatic gene silencing and genome stability to yeast lacking Asf1 [16].

The most obvious difference between yeast and metazoan Asf1 within the histone binding-patch is the S. cerevisiaespecific residue, Ser91. This amino acid defines one end of a large hydrogen-bonding network that runs along the outer-edge of the hydrophobic $\mathrm{H} 3$ binding pocket down to the salt bridge formed by Asf1-Asp54 and H3-Arg129. The centerpiece for this network is a second $S$. cerevisiaespecific residue, Ser48. Oddly, sequence comparisons suggest that this hydrogen-bond network may be less extensive in most eukaryotes: Ser48 is either glycine or alanine, while Ser91 is always a glycine, from fission yeast to man (Figure 3B).

As evidenced by a lack of observable electron density for the side chain atoms of Lys 125 of H3 and Asf1-Arg49, our structure suggests that these $S$. cerevisiae-specific residues do not participate in the histone/chaperone complex (Figure $1 \mathrm{~A}$ ). Lys125 of $\mathrm{H} 3$ is an invariant glutamine in all other species sequenced to date, whereas Arg49 of Asf1 is conserved as either threonine or glutamic acid. Interestingly, NMR studies have identified both Ala48 and Glu49 of HsAsf1a as having two of the largest chemical shift variations upon binding to the C-terminal helix of H3 [20], implicating these residues in the human interaction. It is possible that the conformation of this region may be subtly different in the bound forms of metazoan Asf1, with the corresponding amino acids making different interactions to $\mathrm{H} 3$ as seen for serines 48 and 91. In addition, Gly91 of human Asf1 was also implicated in H3 binding, suggesting that the metazoan Asf1/H3 interaction may have a more hydrophobic character than seen for yeast.

Taken together, these data suggest that both yeast and metazoan Asf1 proteins utilize an element of species-specific interactions to coordinate histone $\mathrm{H} 3$, although the overall conservation of the interface strongly indicates that the global position and orientation of the $\mathrm{H} 3 \alpha 3$ helix are likely to be very similar. This finding raises a cautionary note about the use of proteins from heterologous species in biochemical assays, because small differences in their specific interactions could cause misregulation of Asf1-mediated histone deposition during processes such as DNA replication and transcription.

\section{Structural implications for epigenetic inheritance}

A major question in biology is how cells transmit epigenetic information in the form of histone modifications during passage of the DNA replication fork. Recent data 

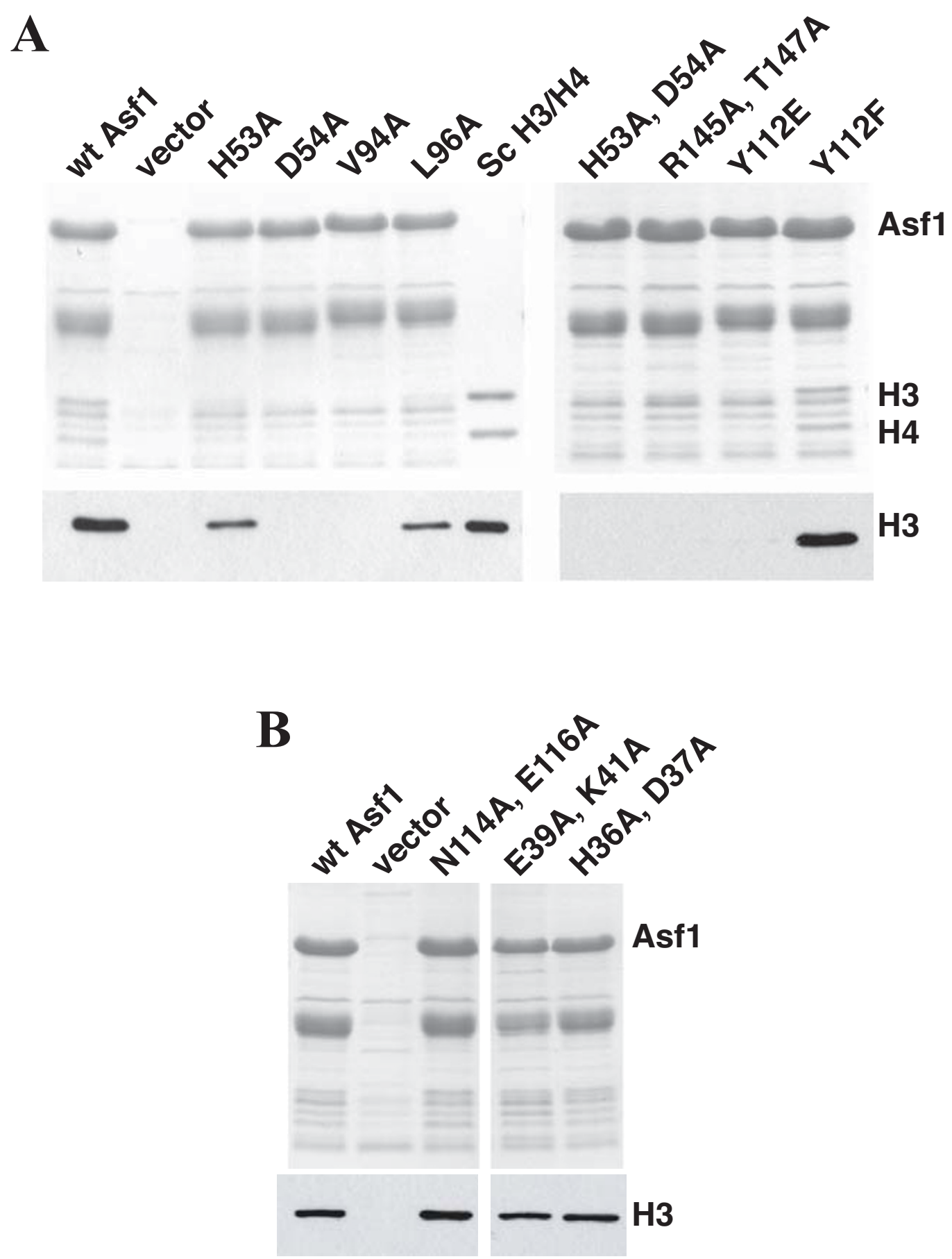

\section{Figure 5}

Biochemical analysis of Asf I residues important for histone binding. (A) Asf I proteins containing mutations in the $\mathrm{H} 3$ binding surface fail to pull-down histones $\mathrm{H} 3 / \mathrm{H} 4$. Extracts were made from $\mathrm{E}$. coli cells expressing yeast histones $\mathrm{H} 3$ and $\mathrm{H} 4$, as well as either wild type or mutant His6-tagged Asfl. Talon metal affinity resin was used to precipitate Asfl and associated proteins. Samples were separated on a I7\% SDS-PAGE gel, visualized by Coomassie staining to indicate recovery of AsfI (upper panel), or analyzed by Western blotting using an anti-H3 antibody (AbCam) (lower panel). Sc $\mathrm{H} 3 / \mathrm{H} 4$ indicates recombinant yeast histones $\mathrm{H} 3 / \mathrm{H} 4$ (0.5 $\mu \mathrm{g}$ upper gel; $10 \mathrm{ng}$ lower gel). (B) Mutation of Asfl residues outside of the histone-binding patch does not disrupt its interaction with histones $\mathrm{H} 3 / \mathrm{H} 4$. The experiment was performed as above. 

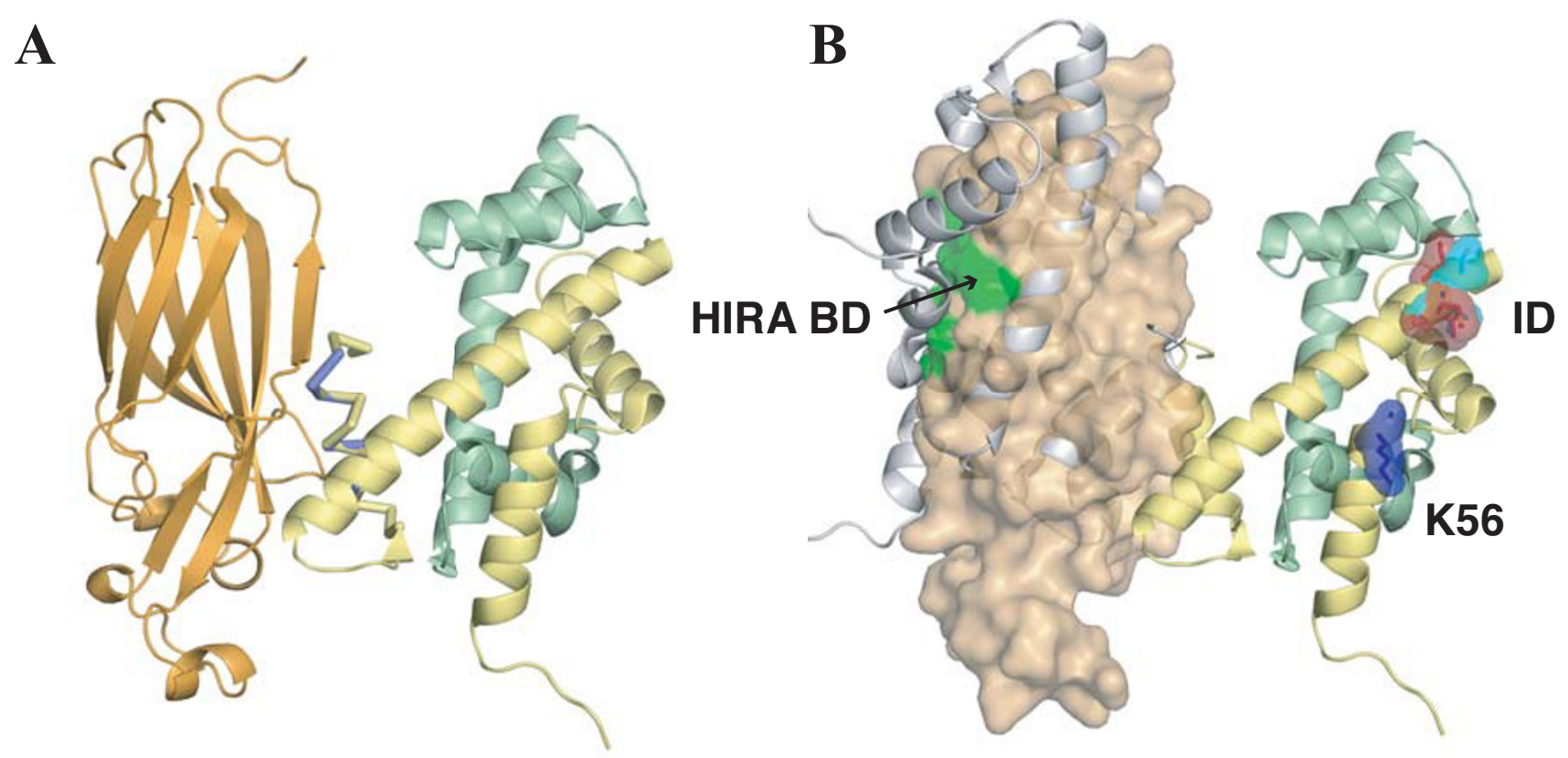

\section{Figure 6}

Model of H3/H4 dimer binding by AsfIN. (A) Asfl binds to a dimer of histones $\mathrm{H} 3 / \mathrm{H} 4$. The structure of AsflN-H3 $\alpha 3$ was modeled onto the structure of the yeast nucleosome (PDB ID IID3) by aligning the $\alpha$-carbons of $\mathrm{H} 3 \alpha 3$ with the respective $\alpha$-carbons of the nucleosome structure. Asfl is shown in orange and is represented in cartoon form. Nucleosomal histones $\mathrm{H} 3$ (yellow) and $\mathrm{H} 4$ (green) are shown in cartoon form with the $\mathrm{H} 3 \mathrm{a} 3$-helix represented as a $\mathrm{C} \alpha$ trace for the nucleosomal (yellow) and complex crystal structure (blue). (B) Asfl occludes binding of a second $\mathrm{H} 3 / \mathrm{H} 4$ dimer while leaving critical decoding residues surface exposed. Asfl is shown with a transparent surface representation. An $\mathrm{H} 3 / \mathrm{H} 4 \mathrm{dimer}$ that would be occluded by Asfl binding is shown as a grey cartoon. Yeast (light blue) and metazoan (red) H3 residues that differ among the $\mathrm{H} 3 . \mathrm{I} / \mathrm{H} 3.2$ and $\mathrm{H} 3.3$ isoforms are shown as sticks with a transparent surface representation, and labeled "ID" for "Isoform Determinants". The H3-K56 residue (dark blue) that is acetylated on newly synthesized histones and the region responsible for HIRA binding (green, labeled "HIRA BD"), are also shown.

have suggested that histone chaperones, including Asf1, bind to $\mathrm{H} 3 / \mathrm{H} 4$ dimers and not tetramers. The existence of $\mathrm{H} 3 / \mathrm{H} 4$ dimers as assembly intermediates raises the possibility that parental dimers can be mixed with nascent ones during replication, forming nucleosomes containing mixed tetramers. This would add an additional layer of complexity to the patterns of inheritance of epigenetic marks on histones during genome duplication [11,24,25].

Our structure suggests a physical model for the control of histone stoichiometry by Asf1. By binding to and occluding proper formation of the four-helix bundle, Asf1 is positioned to directly disrupt histone tetramerization (Figure 6). In this manner, Asf1 may be able to modulate epigenetic information by participating in histone eviction ahead of the replication fork, as is observed during transcription [29], thus promoting mixing of existing and newly-synthesized H3/H4 dimers. Deposition of mixed tetramers would ensure that some daughter nucleosomes contain both parental and nascent histone modifications.

This is a potentially advantageous process, because newly synthesized histone $\mathrm{H} 3$ molecules are acetylated on lysine 56, a modification associated with genome stability and resistance to DNA damaging agents [18,30,31], while parental histones contain epigenetic information critical for the maintenance of a proper transcriptional profile. A future challenge for the field will be to define the mechanisms that form and interpret combinations of modifications on single nucleosomes resulting from $\mathrm{H} 3 / \mathrm{H} 4$ dimer mixing.

\section{Asfl binds to histone $\mathrm{H} 3 / \mathrm{H} 4$ in order to present them to various complexes}

It is becoming clear that a major facet of Asf1's activity is to present histones to other proteins in a specific manner. For example, Asf1 is required for acetylation of newly-syn- 
thesized H3 on K56 prior to deposition by an unidentified enzyme $[18,30]$. Consistent with this finding, our model predicts that H3-K56 is solvent exposed when bound to Asf1 (labeled K56 in Figure 6B).

Additionally, Hake and Allis have proposed an "H3 barcode hypothesis" [9], in which distinct histone isoforms are differentially deposited onto chromatin by interpretation of the their "barcode" through chaperones such as CAF-1 and Hir proteins. Our structural model suggests that Asf1 presents H3/H4 dimers to isoform-specific chaperones in an orientation that could facilitate decoding, allowing the chaperone cooperating with Asf1 to accept or reject a given histone $\mathrm{H} 3$ variant. Consistent with this idea, residues 87-90 of histone $\mathrm{H} 3$, which confer isoform specificity [6], remain exposed following Asf1 binding (labeled "ID" in Figure 6B). Moreover, our model indicates that the HIRA binding site on Asf1 [23] also remains exposed upon histone binding. We propose that Asf1 presents $\mathrm{H} 3$ isoforms to other proteins including CAF-1 and HIRA by forming a ternary structure in which Asf 1 acts as a bridge to stabilize contacts between $\mathrm{H} 3$ and the assisting chaperone. Furthermore, our model predicts that Asf1 would contribute to the histone barcode by generating the proper geometry between histones $\mathrm{H} 3 / \mathrm{H} 4$ and the assisting chaperone in the ternary complex.

\section{Conclusion}

In summary, we have determined the crystal structure of the histone chaperone Asf1 bound to the C-terminal helix of H3. Support for the physiological validity of this structure is provided by genetic as well as biochemical mutational analyses. Significantly, modeling of our structure into the yeast nucleosome explains why Asf1 dissociates histone $\mathrm{H} 3 / \mathrm{H} 4$ tetramers into dimers and suggests that epigenetic inheritance can be directly modulated by Asf1. Future studies will be required to understand the molecular details of histone deposition, the functional synergy between different histone chaperones, and to determine the histone-binding modes of other chaperones.

\section{Methods}

\section{Construct design and purification}

We designed a construct that fused residues 2-155 of Asf1 to the C-terminal alpha helix of histone $\mathrm{H} 3$ (amino acids 121-134) using an eight amino acid linker sequence (AAGAATAA). This construct was cloned into a pET28b derivative containing the sequence for an N-terminal, TEV-cleavable $\mathrm{His}_{6}-\mathrm{MBP}$ tag. The plasmid had been derivatized further to facilitate ligation independent cloning [32]. The fusion protein was purified via Ni-chromatography (Poros-MC, Applied Biosystems), followed by $\mathrm{His}_{6}{ }^{-}$ TEV cleavage of the tag and collection of the flow-through from a second Ni-chromatographic step. Protein was further purified by gel filtration chromatography on a Sepha- rose S-200 column (Amersham Biosciences) and concentrated (Amicon Ultra15, 10 kDa MWCO, Millipore) prior to setting crystallization screens.

\section{Crystallization, data collection, and refinement}

Concentrated protein $(10 \mathrm{mg} / \mathrm{ml})$ was dialyzed against 10 $\mathrm{mM}$ Tris-HCl (pH 7.5), $50 \mathrm{mM} \mathrm{NaCl}$, and $1 \mathrm{mM}$ DTT. Crystals were grown by hanging-drop vapor diffusion by mixing the protein 1:1 with well solution ( $85 \mathrm{mM}$ Tris$\mathrm{HCl}\left(\mathrm{pH} 8.5\right.$ ), $120 \mathrm{mM} \mathrm{Li}_{2} \mathrm{SO}_{4}$, 25\% PEG 4000, and 15\% glycerol) at $18^{\circ} \mathrm{C}$; reservoir solutions were diluted twofold with dialysis buffer prior to sealing crystallization chambers. Crystals were harvested and flash-frozen in liquid nitrogen directly from the drop.

Data were collected using the Advanced Light Source Beamline 8.3.1 at Lawrence Berkeley National Laboratory [33]. Diffraction data were processed using an automated MOSFLM [34] procedure implemented in ELVES [35]. Phases were solved by molecular replacement using Phaser [36] and histone-free yeast Asf1 N-terminal domain as a search model (PDB ID 1ROC) [15]. Model building was carried out using $\mathrm{O}$ [37], and refinement with REFMAC, ARP [38] and CNS [22]. The final model, which contains amino acids 2-155 of ScAsf1 and 121131 of histone $\mathrm{ScH} 3$, has an $\mathrm{R}_{\text {work }}$ of $19.6 \%$ and an $\mathrm{R}_{\text {free }}$ of $23.9 \%$. No density was observed for the fusion linker past the first two alanine residues and the linker was not included in final model. No residues lie in disallowed regions of Ramachandran space. The coordinates and structure factors have been deposited in the Protein Data Bank (PDB ID 2IDC).

\section{E. coli extract histone binding assays}

BL21 (DE3)-Rosetta E.coli cells were transformed with two expression vectors. The first was a polycistronic expression vector [39] that harbors sequences for full-length, untagged yeast histones $\mathrm{H} 3$ and $\mathrm{H} 4$. The second was pET28 (Novagen) expressing $\mathrm{His}_{6}$-tagged wild type or mutant Asf1 $[13,18]$, or an empty vector. Protein expression was induced at $\mathrm{A}_{600}=0.5-0.6$ at $30^{\circ} \mathrm{C}$ for 5 hours with $1 \mathrm{mM}$ IPTG. Cell extracts were made by sonication in a Lysis Buffer containing $20 \mathrm{mM}$ Tris-Cl (pH 7.5), 500 $\mathrm{mM} \mathrm{NaCl}, 0.01 \% \mathrm{NP} 40,10 \mathrm{mM}$ imidazole and a cocktail of protease inhibitors. $4 \mathrm{mg}$ of soluble extract protein was incubated at $4{ }^{\circ} \mathrm{C}$ for $2 \mathrm{hr}$ with $\sim 20 \mu \mathrm{l}$ of Talon metal affinity resin (Clonetech). Beads were recovered by centrifugation and washed three times with $1 \mathrm{ml}$ of Lysis Buffer for 10 minutes. Precipitated proteins were eluted with SDSPAGE sample buffer and visualized by Coomassie G stain of a $17 \%$ SDS-PAGE gel. Histone H3 was detected by western blot using an $\alpha-\mathrm{H} 3$ polyclonal antibody (AbCam). 


\section{Telomeric silencing assay}

The silencing assay and strain containing asf $1 \Delta, \operatorname{cac} 1 \Delta$, and URA3-VIIL alleles has been described previously [13]

\section{Authors' contributions}

AJA prepared the Asf1/H3 fusion protein, generated crystals, and collected the diffraction data. AJA solved the structure, and together with JMB inspected the final model and parameters for accuracy. TT performed the Asf1 mutagenesis, histone binding and silencing experiments. AJA, PDK and JMB designed and initiated the experiments. AJA wrote the manuscript with revisions from JMB and PDK. All authors read and approved the final manuscript.

\section{Acknowledgements}

We thank James Holton, George Meigs and Jane Tanamachi for assistance at ALS Beamline 8.3.I. We also thank Emmanuel Skordalakes and Kevin

Corbett for their invaluable assistance and expertise. This work was sup- ported by grants from the NSF (MCB-0549I3I, PDK) and the NCl (CA077373, JMB). Data and coordinates have been submitted to the Protein Data Bank as PDB ID 2IDC.

Note: While this paper was under review, a paper by Tyler and colleagues was published describing the interaction of Asfl with histones $\mathrm{H} 3 / \mathrm{H} 4$ (English et al., Cell, 2006). This work confirms that the orientation of our $\mathrm{H} 3$ peptide is correct and validates our modeling for Asfl-dependent regulation of $\mathrm{H} 3 / \mathrm{H} 4$ stoichiometry.

\section{References}

I. Woodcock CL: Chromatin architecture. Curr Opin Struct Biol 2006, 16(2):213-220.

2. Shibahara K, Stillman B: Replication-dependent marking of DNA by PCNA facilitates CAF-I-coupled inheritance of chromatin. Cell 1999, 96(4):575-585.

3. Ray-Gallet D, Quivy JP, Scamps C, Martini EM, Lipinski M, Almouzni G: HIRA is critical for a nucleosome assembly pathway independent of DNA synthesis. Mol Cell 2002, 9(5): 1091 - I I00.

4. Green EM, Antczak AJ, Bailey AO, Franco AA, Wu KJ, Yates JR 3rd, Kaufman PD: Replication-independent histone deposition by the HIR complex and Asfl. Curr Biol 2005, I5(22):2044-2049.

Table I: Summary of Crystallographic Statistics

\begin{tabular}{|c|c|}
\hline Data Collection & \\
\hline Radiation $\AA$ & 1.11587 \\
\hline Space Group & $P 2,2,2$, \\
\hline \multirow[t]{3}{*}{ Unit Cell Dimensions $\AA$} & $\mathrm{a}=44.43$ \\
\hline & $b=52.09$ \\
\hline & $c=86.68$ \\
\hline Resolution $\AA$ & $52-2.1$ \\
\hline Completeness \% (last shell) & $98.7(98.7)$ \\
\hline Rsym \% (last shell) & $6(36.2)$ \\
\hline Multiplicity (last shell) & $3.9(3.9)$ \\
\hline Overall I/s (last shell) & $9.1(2.0)$ \\
\hline Wilson B factor & 35.08 \\
\hline \multicolumn{2}{|l|}{ Molecular Replacement } \\
\hline Search Model & PDB ID IROC \\
\hline $\mathrm{R}$ factor* & 32.2 \\
\hline Correlation coefficient* & 0.876 \\
\hline \multicolumn{2}{|l|}{ Structural Refinement } \\
\hline Resolution Å (last bin) & $30-2.2(2.257-2.20)$ \\
\hline Number of nonhydrogen atoms & 1429 \\
\hline Munber of protein atoms & 1338 \\
\hline Number of water atoms & 91 \\
\hline Overall reflections (last shell) & $9995(720)$ \\
\hline Test reflections (last shell) & $500(42)$ \\
\hline $\mathrm{R}_{\text {work }} / \mathrm{R}_{\text {free }} \%$ & $19.6 / 23.9$ \\
\hline Rmsd $_{\text {bonds/angles }}$ & $0.017 / 1.614$ \\
\hline \multicolumn{2}{|l|}{ Ramachandran \% (No. of residues) } \\
\hline Most favored & $99.39(162)$ \\
\hline Allowed & $0.61(1)$ \\
\hline \multicolumn{2}{|l|}{ Average $B$ factors } \\
\hline Protein & 55.87 \\
\hline Water & 62.41 \\
\hline
\end{tabular}

*After one round of refinement in Refmac. 
5. Prochasson P, Florens L, Swanson SK, Washburn MP, Workman JL: The HIR corepressor complex binds to nucleosomes generating a distinct protein/DNA complex resistant to remodeling by SWI/SNF. Genes Dev 2005, 19(2I):2534-2539.

6. Ahmad K, Henikoff S: The histone variant H3.3 marks active chromatin by replication-independent nucleosome assembly. Mol Cell 2002, 9(6): | |9|-I200.

7. Loppin B, Bonnefoy E, Anselme C, Laurencon A, Karr TL, Couble P The histone $\mathrm{H} 3.3$ chaperone HIRA is essential for chromatin assembly in the male pronucleus. Nature 2005, 437(7063): $1386-1390$.

8. van der Heijden GW, Dieker JW, Derijck AA, Muller S, Berden JH, Braat DD, van der Vlag J, de Boer P: Asymmetry in histone H3 variants and lysine methylation between paternal and maternal chromatin of the early mouse zygote. Mech Dev 2005, I 22(9): 1008-1022.

9. Hake $S B$, Allis $C D$ : Histone $\mathbf{H 3}$ variants and their potential role in indexing mammalian genomes: the " $\mathrm{H} 3$ barcode hypothesis". Proc Natl Acad Sci U S A 2006, 103 ( I 7):6428-6435.

10. Wu RS, Bonner WM: Separation of basal histone synthesis from S-phase histone synthesis in dividing cells. Cell I98I, 27(2 pt I):32I-330.

II. Tagami H, Ray-Gallet D, Almouzni G, Nakatani Y: Histone H3.I and H3.3 complexes mediate nucleosome assembly pathways dependent or independent of DNA synthesis. Cell 2004, I | 6(I):5|-6|.

12. Tyler JK, Collins KA, Prasad-Sinha J, Amiott E, Bulger M, Harte PJ, Kobayashi R, Kadonaga JT: Interaction between the Drosophila CAF-I and ASFI chromatin assembly factors. Mol Cell Biol 200I, 2 I(19):6574-6584.

13. Sharp JA, Fouts ET, Krawitz DC, Kaufman PD: Yeast histone deposition protein Asflp requires Hir proteins and PCNA for heterochromatic silencing. Curr Biol 200 I, I I(7):463-473.

14. Tyler JK, Adams CR, Chen SR, Kobayashi R, Kamakaka RT, Kadonaga JT: The RCAF complex mediates chromatin assembly during DNA replication and repair. Nature 1999, 402(676I):555-560.

15. Daganzo SM, Erzberger JP, Lam WM, Skordalakes E, Zhang R, Franco AA, Brill SJ, Adams PD, Berger JM, Kaufman PD: Structure and function of the conserved core of histone deposition protein Asfl. Curr Biol 2003, I3(24):2। 48-2I 58.

16. Tamburini BA, Carson JJ, Adkins MW, Tyler JK: Functional conservation and specialization among eukaryotic anti-silencing function I histone chaperones. Eukaryot Cell 2005, 4(9): $1583-1590$

17. Franco AA, Lam WM, Burgers PM, Kaufman PD: Histone deposition protein AsfI maintains DNA replisome integrity and interacts with replication factor C. Genes Dev 2005, I (I I ): I365-I375.

18. Recht J, Tsubota T, Tanny JC, Diaz RL, Berger JM, Zhang X, Garcia BA, Shabanowitz J, Burlingame AL, Hunt DF, et al.: Histone chaperone Asfl is required for histone $\mathrm{H} 3$ lysine $\mathbf{5 6}$ acetylation, a modification associated with $\mathbf{S}$ phase in mitosis and meiosis. Proc Natl Acad Sci U S A 2006, 103( I 8):6988-6993.

19. Umehara T, Chimura T, Ichikawa N, Horikoshi M: Polyanionic stretch-deleted histone chaperone cial/Asflp is functional both in vivo and in vitro. Genes Cells 2002, 7(I):59-73.

20. Mousson F, Lautrette A, Thuret JY, Agez M, Courbeyrette R, Amigues B, Becker E, Neumann JM, Guerois R, Mann C, et al.: Structural basis for the interaction of Asfl with histone $\mathrm{H} 3$ and its functional implications. Proc Natl Acad Sci U S A 2005, I 02( I 7):5975-5980

21. Munakata T, Adachi N, Yokoyama N, Kuzuhara T, Horikoshi M: A human homologue of yeast anti-silencing factor has histone chaperone activity. Genes Cells 2000, 5(3):22I-233.

22. Brunger AT, Adams PD, Clore GM, DeLano WL, Gros P, GrosseKunstleve RW, Jiang JS, Kuszewski J, Nilges M, Pannu NS, et al.: Crystallography \& NMR system: A new software suite for macromolecular structure determination. Acta Crystallogr D Biol Crystallogr 1998, 54(Pt 5):905-921.

23. Zhang R, Poustovoitov MV, Ye X, Santos HA, Chen W, Daganzo SM, Erzberger JP, Serebriiskii IG, Canutescu AA, Dunbrack RL, et al.: Formation of MacroH2A-containing senescence-associated heterochromatin foci and senescence driven by ASFIa and HIRA. Dev Cell 2005, 8(I):19-30.

24. Annunziato AT: Split decision: what happens to nucleosomes during DNA replication? J Biol Chem 2005, 280(13): I2065-I 2068.
25. English CM, Maluf NK, Tripet B, Churchill ME, Tyler JK: ASFI binds to a heterodimer of histones $\mathrm{H} 3$ and $\mathrm{H} 4$ : a two-step mechanism for the assembly of the $\mathrm{H} 3-\mathrm{H} 4$ heterotetramer on DNA. Biochemistry 2005, 44(42): | 3673-| 3682

26. Banks DD, Gloss LM: Folding mechanism of the (H3-H4)2 histone tetramer of the core nucleosome. Protein Sci 2004 I3(5): | 304-1316.

27. Eickbush TH, Moudrianakis EN: The histone core complex: an octamer assembled by two sets of protein-protein interactions. Biochemistry 1978, I7(23):4955-4964.

28. White CL, Suto RK, Luger K: Structure of the yeast nucleosome core particle reveals fundamental changes in internucleosome interactions. Embo J 200I, 20( I 8):5207-52I8.

29. Schwabish MA, Struhl K: Asfl mediates histone eviction and deposition during elongation by RNA polymerase II. Mol Cell 2006, 22(3):4I5-422.

30. Celic I, Masumoto H, Griffith WP, Meluh P, Cotter RJ, Boeke JD, Verreault $A$ : The sirtuins hst 3 and Hst4p preserve genome integrity by controlling histone $\mathbf{h}$ lysine $\mathbf{5 6}$ deacetylation. Curr Biol 2006, I 6(I3): I280-1289.

31. Masumoto H, Hawke D, Kobayashi R, Verreault A: A role for cellcycle-regulated histone $\mathrm{H} 3$ lysine 56 acetylation in the DNA damage response. Nature 2005, 436(7048):294-298.

32. Li C, Evans RM: Ligation independent cloning irrespective of restriction site compatibility. Nucleic Acids Res 1997. 25(20):4|65-4I66.

33. MacDowell AA, Celestre RS, Howells M, McKinney W, Krupnick J, Cambie D, Domning EE, Duarte RM, Kelez N, Plate DW, et al.: Suite of three protein crystallography beamlines with single superconducting bend magnet as the source. I Synchrotron Radiat 2004, I I (Pt 6):447-455

34. Powell HR: The Rossmann Fourier autoindexing algorithm in MOSFLM. Acta Crystallogr D Biol Crystallogr 1999, 55(Pt 10):1690-1695.

35. Holton J, Alber T: Automated protein crystal structure determination using ELVES. Proc Natl Acad Sci U S A 2004, I0I(6): I537-I542.

36. Read RJ: Pushing the boundaries of molecular replacement with maximum likelihood. Acta Crystallogr D Biol Crystallogr 200I, 57(Pt I0): I373-1382.

37. Jones TA, Zou JY, Cowan SW, Kjeldgaard : Improved methods for building protein models in electron density maps and the location of errors in these models. Acta Crystallogr A 1991, 47(Pt 2):II0-119.

38. Winn MD, Isupov MN, Murshudov GN: Use of TLS parameters to model anisotropic displacements in macromolecular refinement. Acta Crystallogr D Biol Crystallogr 200I, 57(Pt I): $122-133$.

39. Tan S: A modular polycistronic expression system for overexpressing protein complexes in Escherichia coli. Protein Expr Purif 200I, 2I(I):224-234.

Publish with Biomed Central and every scientist can read your work free of charge

"BioMed Central will be the most significant development for disseminating the results of biomedical research in our lifetime. "

Sir Paul Nurse, Cancer Research UK

Your research papers will be:

- available free of charge to the entire biomedical community

- peer reviewed and published immediately upon acceptance

- cited in PubMed and archived on PubMed Central

- yours - you keep the copyright

Submit your manuscript here:

http://www.biomedcentral.com/info/publishing_adv.asp
BioMedcentral 